\title{
BMC Medical Ethics reviewer acknowledgement 2014
}

\section{Clare Partridge}

\section{Contributing reviewers}

The editors of BMC Medical Ethics would like to thank all our reviewers who have contributed to the journal in Volume 15 (2014).

$\begin{array}{lll}\text { Aslihan Akpinar } & \text { Carol Ashton } & \text { Charlyn Black } \\ \text { Turkey } & \text { USA } & \text { Canada } \\ \text { Eman Al Gaai } & \text { Peter John Aspinall } & \text { Isra Black } \\ \text { Saudi Arabia } & \text { UK } & \text { UK } \\ \text { Amel Alghrani } & \text { Jacquineau Azetsop } & \text { Fiona Boland } \\ \text { UK } & \text { Chad } & \text { Ireland } \\ \text { Afshan Ali } & \text { Francis Barchi } & \text { Roseanna Bourke } \\ \text { Saudi Arabia } & \text { USA } & \text { New Zealand } \\ \text { M. Z. Alkawi } & \text { Lianne Barnieh } & \text { Deborah Bowen } \\ \text { Saudi Arabia } & \text { Canada } & \text { USA } \\ \text { James A Anderson } & \text { Drue Barrett } & \text { Marek Brabec } \\ \text { Canada } & \text { USA } & \text { Czech Republic } \\ \text { Melissa Anderson } & \text { Philip Bejon } & \text { Iain Brassington } \\ \text { USA } & \text { Kenya } & \text { UK } \\ \text { Chike Anibeze } & \text { Solomon (Solly) Benatar } & \text { Gina Bravo } \\ \text { Nigeria } & \text { South Africa } & \text { Canada } \\ \text { Marco Annoni } & \text { Sara Bergstresser } & \text { Niamh Brennan } \\ \text { Italy } & \text { USA } & \text { Ireland } \\ \text { Paul Appelbaum } & \text { Guido Biasco } & \text { Howard Brody } \\ \text { USA } & \text { Italy } & \text { USA } \\ \text { Julie Armin } & \text { Ariella Binik } & \text { Kyle Brothers } \\ \text { USA } & \text { UK } & \text { USA } \\ \text { Atsushi Asai } & \text { Giles Birchley } & \text { Hilde Buiting } \\ \text { Japan } & \text { UK } & \text { Netherlands } \\ \text { Richard Ashcroft } & \text { Felicity Bishop } & \text { Eric Campbell } \\ \text { UK } & \text { UK } & \text { USA }\end{array}$

Correspondence: clare.partridge@biomedcentral.com

BioMed Central, Floor 6, 236 Gray's Inn Road, London WC1X 8HB, UK 
Krysia Canvin

UK

Adriana Ceci
Italy

Kenneth Chambaere

Belgium

Audrey Chapman

USA

Kath Checkland

UK

Lei-Shih Chen

USA

Gabrielle Christenhusz

Belgium

Murat Civaner

Turkey

Ellen Clayton

USA

Adam Cohen

Netherlands

Joachim Cohen

Belgium

Luana Colloca

USA

James Colquitt

Armenia

Andrew Courtwright

USA

John Coverdale

USA

Andrew Crowden

Australia

Rafael Dal-Ré

Spain

Bege Dauda

Belgium

Angus Dawson

UK

Liza Dawson

USA

Jacques Demotes

France

Ioannis Dimoliatis

Greece
Edward Dove

Canada

Anne-Elisabeth Driessen

Netherlands

Brian Earp

UK

Marleen Eijkholt

USA

Susan Ellenberg

USA

Nathan Emmerich

UK

Miran Epstein

UK

Daniele Fanelli

UK

Conrad Fernandez

Canada

Joel Frader

USA

Samuel Frank

USA

Lucy Frith

UK

Davina Ghersi

Australia

Nader Ghotbi

Japan

Noor Giesbertz

Netherlands

Simona Giordano

UK

Alberto Giubilini

Australia

Aaron Goldenberg

USA

Joseph Graves Jr.

USA

Kalle Grill

Sweden

Thomas Grisso

USA

Ronella Grootens-Wiegers

Netherlands
Marilys Guillemin

Australia

Tolga Guven

Turkey

Bridget Haire

Australia

Arja Halkoaho

Finland

Muhammad Hammami

Saudi Arabia

Sumaya Hammami

USA

Martyn Hammersley

UK

Wolfgang Hampe

Germany

Sven Ove Hansson

Sweden

Gert Helgesson

Sweden

Kristien Hens

Netherlands

Jonathan Herring

UK

Spencer Hey

Canada

Soren Holm

UK

Neil A. Holtzman

USA

Matthew Hotopf

UK

Heidi C Howard

Sweden

Sara Hull

USA

Matthew Hunt

Canada

Richard Huxtable

UK

Rick Iedema

Australia

Carel Ijsselmuiden

Switzerland 
Julia Inthorn

Germany

Saima Iqbal

Pakistan

Jonathan Ives

UK

Simon Jenkins

UK

Steven Joffe

USA

Yann Joly

Canada

Karsten Juhl Jørgensen

Denmark

Ralf Jox

Germany

Niklas Juth

Sweden

Gernot Kaiser

Germany

Dorcas Kamuya

Kenya

Hitomi Kataoka

Japan

Georg Kemmler

Austria

Melissa Kendall

Australia

Jack Kennelly

New Zealand

Ian Kerridge

Australia

Sy Kim

USA

Sharon Kling

South Africa

Sheldon Krimsky

USA

Lisa Lee

USA

Simon J. Craddock Lee

USA

Penney Lewis

UK
Charles Lidz

USA

Geoffrey Lomax

USA

Jose-Antonio Lopez-Pina

Spain

Sana Loue

USA

Jayne Lucke

Australia

Tamra Lysaght

Singapore

Kristin Bakke Lysdahl

Norway

Graeme Maclaren

Singapore

Shoichi Maeda

Japan

Morten Magelssen

Norway

Nicola Magnavita

Italy

Aisha Malik

UK

Phillipa Malpas

New Zealand

Vicki Marsh

Kenya

Deborah Mascalzoni

Italy

Bojana Matejic

Serbia

Sheryl McCurdy

USA

Martin McKneally

Canada

Robert McMaster

UK

Lindsay McNair

USA

Darlene McNaughton

Australia

Andrew McRae

Canada
Marcel Mertz

Germany

Kenneth Miller

USA

Francesca Minerva

Australia

Bert Molewijk

Netherlands

Sassy Molyneux

Kenya

Keymanthri Moodley

South Africa

Paul Mueller

USA

Paul Ndebele

Zimbabwe

Peter A Newman

Canada

Dianne Nicol

Australia

Erik Nord

Norway

Susan L Norris

USA

David Obree

UK

Elke Birgit Ochsmann

Germany

Anke Oerlemans

Netherlands

Sadayoshi Ohbu

Japan

Kanu Okike

USA

Taketoshi Okita

Japan

Stuart Oultram

UK

John Owens

UK

Vural Ozdemir

Canada

Bennett Pafford

USA 
Vassilios Papalois

UK

Michael Parker

UK

John Porter

UK

Corinna Porteri

Italy

Rouven Christian Porz

Switzerland

Stefan Priebe

UK

Marta Pulido

Spain

Divya Rajaraman

India

Flavia Ramos

Brazil

Stella Reiter-Theil

Switzerland

Emmanuelle Rial-Sebbag

France

Christopher Robertson

USA

Eduardo Rodriguez

Chile

Wendy Rogers

Australia

Lainie Ross

USA

Theresa Rossouw

South Africa

Mark Rothstein

USA

Michael Rowe

USA

Sabine Salloch

Germany

Lars Sandman

Sweden

Pamela Sankar

USA

Julian Savulescu

UK
Jerome Schofferman

USA

Sarah Hudson Scholle

USA

Vera Scott

South Africa

Clive Seale

UK

Janet Seeley

Uganda

Mary Seeman

Canada

Nayha Sethi

UK

Jane Seymour

UK

Julian Sheather

UK

Diego Silva

Canada

Jerome Singh

South Africa

Catherine Slack

South Africa

Elpidoforos Soteriades

Cyprus

Jocelyne St-Arnaud

Canada

Ruth Stirton

UK

Erwin Stolz

Austria

Daniel Strech

Germany

Mia Svantesson

Sweden

Elisabeth Svensson

Sweden

Leslie Swartz

South Africa

Emmanouil Symvoulakis

Greece

Anne-Marie Tassé

Canada
Patrick Taylor

USA

Sally Theobald

UK

Engelbert Theurl

Austria

Paulina Tindana

Ghana

Gustav Tinghög

Sweden

Tsuen-Chiuan Tsai

Taiwan

Kristen Underhill

USA

Johannes Van Delden

Netherlands

Rieke Van Der Graaf

Netherlands

Joseph Verheijde

USA

A.M. Viens

UK

Julia Wade

UK

Elizabeth Wager

UK

Rebecca Walker

USA

James Warner

UK

Douglas Wassenaar

South Africa

Robert Wheeler

UK

Karolyn White

Australia

David Kenneth Whynes

UK

Guy Widdershoven

Netherlands

Garrath Williams

UK

Bryn Williams-Jones

Canada 
Erica Witkamp

Netherlands

Amanda Wolf

New Zealand

Luke Wolfenden

Australia

Heiner Wolters

Germany
Simon Woods

UK

Christina Zampas

Sweden

Ma'N H. Zawati

Canada

Deborah Zion

Australia

\section{Daniella Zipkin}

USA

\section{Matjaz Zwitter}

Slovenia

Received: 7 January 2015 Accepted: 7 January 2015 Published: 10 February 2015

doi:10.1186/1472-6939-16-5

Cite this article as: Partridge: BMC Medical

Ethics reviewer acknowledgement 2014. BMC Medical Ethics 2015 16:5. 